\title{
Correction to: Pharmacokinetic and Tissue Distribution Profile of Long Acting Tenofovir Alafenamide and Elvitegravir Loaded Nanoparticles in Humanized Mice Model
}

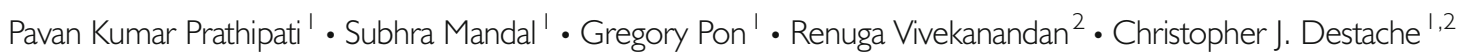

Published online: 20 May 2019

(C) Springer Science+Business Media, LLC, part of Springer Nature 2019

\section{Correction to: Pharm Res (2017) 34:2749-2755 https://doi.org/ I0.1007/s I 1095-017-2255-7}

There are errors in the published article (Volume 34, Number 12, pp. 2749-2755).

The errors occurred in pharmacokinetic study design of materials and methods section on page 2750-51.

1. Another group of 15 mice ( 3 mice/time point) were injected SubQ with $200 \mathrm{mg} / \mathrm{kg}$ (each TAF, FTC, and EVG) of free drug in $1 \mathrm{~mL}$ of $5 \%$ dextrose.

FTC is not related to the objective of this article and it has no reported drug-drug interactions that could affect PK of TAF and EVG [1].

\section{Refererence}

1. AIDSinfo, Drug interactions between nucleoside reverse transcriptase inhibitors and other drugs (including antiretroviral agents). in: U.S.D.o.H.H. Services (Ed.), U.S. Department of Health \& human Services, Washington, DC, USA, 2018.

2. Institutional animal care and use committee approved protocol (\#0989) and procedures were followed. However, the authors retrospectively found that "injecting naïve TAF+ FTC+EVG solution to mice" was not in the approved protocol.

Publisher's Note Springer Nature remains neutral with regard to jurisdictional claims in published maps and institutional affiliations.

The online version of the original article can be found at $h$ ttps://doi.org//0.1007/ sl 1095-0|7-2255-7

Pavan Kumar Prathipati

Pavankumarprathipati@creighton.edu

Pharmacy Practice Department, Creighton University School of Pharmacy \& Health Professions, 2500, California Plaza, Omaha, NE 68178, USA

2 School of Medicine, Creighton University, Omaha, NE, USA 